\title{
PCSK9 Inhibitors: Clinical Relevance, Molecular Mechanisms, and Safety in Clinical Practice
}

\author{
Filipe Ferrari, ${ }^{1,2}$ Ricardo Stein, ${ }^{1,2,3,4,6}$ Marcelo Trotte Motta, ${ }^{5 \odot}$ Emilio Hideyuki Moriguchi, ${ }^{1,6,7 \odot}$ \\ Programa de Pós-Graduação em Cardiologia e Ciências Cardiovasculares - Hospital de Clínicas de Porto Alegre (HCPA) - Universidade Federal \\ do Rio Grande do Sul (UFRGS), ${ }^{1}$ Porto Alegre, RS - Brazil \\ Grupo de Pesquisa em Cardiologia do Exercício (CardioEx) - Hospital de Clínicas de Porto Alegre (HCPA) - Universidade Federal do Rio Grande \\ do Sul (UFRGS), ${ }^{2}$ Porto Alegre, RS - Brazil \\ Faculdade de Medicina - Hospital de Clínicas de Porto Alegre - Universidade Federal do Rio Grande do Sul (UFRGS), ${ }^{3}$ Porto Alegre, RS - Brazil \\ Vitta Centro de Bem-Estar Físico, ${ }^{4}$ Porto Alegre, RS - Brazil \\ Universidade Estadual de Feira de Santana, ${ }^{5}$ Feira de Santana, BA - Brazil \\ Divisão de Medicina Interna - Hospital de Clínicas de Porto Alegre, ${ }^{6}$ Porto Alegre, RS - Brazil \\ Departamento de Medicina Interna - Escola de Medicina - Universidade Federal do Rio Grande do Sul (UFRGS), ${ }^{7}$ Porto Alegre, RS - Brazil
}

\section{Abstract}

Coronary artery disease (CAD) is one of the leading causes of mortality. High circulating levels of low-density lipoprotein (LDL) in the blood are associated with cardiovascular mortality, whether through an etiological role or through its association with the progression of CAD per se. Randomized clinical trials have shown that, when LDL levels are reduced, cardiovascular risk is also reduced, which reinforces this association. The first major trial involving a hypolipidemic agent of the statin family, the Scandinavian Simvastatin Survival Study (4S), was published in 1994 and found a significant reduction in mortality in patients at high cardiovascular risk. However, even in subsequent studies with different statins, a residual risk persisted, and this seems not to have changed over time; it is speculated that this risk may be due to statin intolerance. In this scenario, the potential exists for novel hypolipidemic agents to drive a true revolution in the therapy of dyslipidemia. The recent discovery of PCSK9 inhibitors (PCSK9i), a class of hypolipidemic monoclonal antibodies, is extremely promising. PCSK9 inhibition is capable of promoting a mean LDL reduction of up to $60 \%$, with potential for very significant clinical repercussions, as every $38 \mathrm{mg} / \mathrm{dL}$ reduction in LDL appears to be associated with a $22 \%$ reduction in cardiovascular risk. This review addresses a brief history of PCSK9i, major trials of these drugs, cardiovascular outcomes, and aspects related to their efficacy and safety. Finally, the molecular mechanisms and possible pleiotropic effects of PCSK9i are also discussed.

\section{Introduction}

Worldwide, cardiovascular diseases account for almost half of all deaths in people under 70. In Brazil, they were responsible

\section{Keywords}

Cardiovascular Diseases/physiopathology; Coronary Artery Disease/mortality; Proprotein Convertase 9; Cholesterol, LDL; Lipoproteins; Anticholesteromic Agents.

\footnotetext{
Mailing Address: Emilio Hideyuki Moriguchi •

Universidade Federal do Rio Grande do Sul - Av. Paulo Gama, 110. Postal

Code 90040-060, Farroupilha, Porto Alegre, RS - Brazil

E-mail: emoriguchi@hcpa.edu.br

Manuscript received September 23, 2018, revised manuscript October 28 2018, accepted November 01, 2018
}

DOI: 10.5935/abc.20190029 for almost $30 \%$ of deaths in $2013 .{ }^{1}$ In recent decades, mounting evidence has shown a close link between low-density lipoprotein (LDL) levels and incidence of coronary artery disease (CAD)..$^{2,3}$ Inadequate hepatic uptake of LDL results in increased levels of circulating LDL, and consequent incidence of premature CAD. ${ }^{4}$

The treatment of dyslipidemias involves a number of factors, and lifestyle changes should be part of all medical prescriptions for this purpose. Non-pharmacological interventions, such as starting a regular exercise program, not smoking or quitting smoking, and adopting a healthy diet can have a significant impact on lipid profile. However, a substantial number of patients need to add hypolipidemic drugs (e.g., statins, ezetimibe, fibrates) to the aforementioned measures to achieve recommended LDL goals. ${ }^{5}$

Substantial advances in lipid-lowering drugs have been achieved in recent years. ${ }^{6}$ When used appropriately, these agents play a preponderant role in preventing adverse cardiovascular (CV) outcomes. ${ }^{7}$ Hypolipidemic therapy with statins has been shown to have an impact both for primary prevention of atherosclerosis in patients at high $\mathrm{CV}$ risk $^{8}$ and for secondary prevention. However, some patients do not reach desired LDL levels even at maximal doses of statins (whether as monotherapy or up to triple therapy) or even when ezetimibe is added to statin therapy; this results in an important residual risk of CV events. ${ }^{9,10}$ Thus, the search for therapeutic alternatives that can reduce LDL more aggressively, aiming to achieve better outcomes, continues.

Among recent developments, perhaps the most outstanding class of novel lipid-lowering agents are the proprotein convertase subtilisin/kexin type 9 inhibitors (PCSK9i). ${ }^{11}$ PCSK9 is a protein that ultimately promotes the degradation of hepatic LDL receptors, leading to hypercholesterolemia. ${ }^{6,7}$ The PCSK9i are monoclonal antibodies that increase the availability of LDL receptors. When PCSK9 is inhibited, there is greater uptake of LDL by their respective receptors present in hepatocytes, with reduction of serum and plasma levels of LDL (Figure 1). ${ }^{12,13}$

An important point about the causal relationship between LDL levels and CV outcomes is the dose-response behavior observed. To the extent that high LDL levels increase CV risk, when LDL levels are reduced, so is the rate of adverse CV outcomes. For example, the JUPITER study demonstrated that use of a statin (rosuvastatin) for 2 years was able to protect patients substantially, especially in those with LDL levels were below $45 \mathrm{mg} / \mathrm{dL}{ }^{14}$ 


\section{Clinical relevance}

$\mathrm{CV}$ risk can be significantly mitigated by aggressive LDL reductions; the higher the risk, the lower the target LDL level. No class of hypolipidemic agents had given rise to such anticipation since the discovery of the statins, ${ }^{15}$ as the PCSK9i can promote an additional reduction of up to $60 \%$ in LDL levels when compared to statins. ${ }^{9}$

The FOURIER study, ${ }^{8}$ a randomized clinical trial (RCT) published in 2017, enrolled more than 27,000 patients with atherosclerotic CV disease and LDL levels $\geq 70 \mathrm{mg} / \mathrm{dL}$. Participants, all of whom were on statin therapy, were randomly allocated to receive add-on evolocumab or placebo for a mean period of 2.2 years. In the evolocumab group, there was a mean reduction in LDL levels of $30 \mathrm{mg} / \mathrm{dL}$ from baseline; in absolute terms, when compared to the placebo group, the mean LDL reduction was $56 \mathrm{mg} / \mathrm{dL}$. Most importantly, a 15\% reduction was found in the primary endpoint (nonfatal acute myocardial infarction [AMI], stroke, coronary revascularization, hospitalization for unstable angina, and CV mortality), as well as a $20 \%$ reduction in the composite secondary hard endpoint of $\mathrm{CV}$ death, nonfatal $\mathrm{AMI}$, and nonfatal stroke. At the end of the study, there was an absolute risk reduction of $1.5 \%$ for both the primary and secondary endpoints, which translated into a number needed to treat (NNT) of approximately 67 .

More recently, the ODYSSEY Outcomes ${ }^{16}$ study compared alirocumab plus with statin versus statin alone at maximal tolerated dose in approximately 19,000 patients at very high CV risk for 2.8 years. LDL levels were $53.3 \mathrm{mg} / \mathrm{dL}$ in the alirocumab + statin group versus $101.4 \mathrm{mg} / \mathrm{dL}$ in the statin group, and an absolute reduction of $54.7 \%$ was observed. The primary outcome of major adverse CV events was also significantly lower in the combination therapy group. Furthermore, there was a surprising 15\% reduction in deaths from any cause in this group (NNT of approximately 63). In the ODYSSEY Outcomes trial, LDL decreased $47 \mathrm{mg} / \mathrm{dL}$ after 1 year of follow-up, which, based on the Cholesterol Treatment Trialists (CTT) model, ${ }^{17}$ would represent a $24 \%$ reduction in the relative risk of major CV events. However, in practice, only a $15 \%$ reduction was observed. This divergence can be explained by the difference in follow-up time between ODYSSEY Outcomes (2.5 years) and the CCT analyses (5 years). In fact, CTT data showed a smaller magnitude of benefit regarding LDL reduction in the first year. ${ }^{17}$

In an analysis of the FOURIER trial, ${ }^{18}$ the clinical benefits of evolocumab differed interestingly depending on the severity and extent of CAD. First, evolocumab reduced LDL levels by $61 \%$. Second, patients with a greater risk profile, i.e., those with more recent $\mathrm{AMI}$ ( $<2$ years), multiple anterior AMIs, and multivessel disease, were those who benefited most from the use of PCSK9i: they experienced relative risk reductions for the primary endpoint of $20 \%, 18 \%$, and $21 \%$, respectively, versus $5 \%, 8 \%$, and $7 \%$ reductions respectively in low-risk comparators subgroups (i.e., participants without these complications). In the high-risk patient subgroups, the absolute risk reductions in 3 years exceeded $3 \%(3.4 \%$, $3.7 \%$, and $3.6 \%$ respectively), versus approximately $1 \%$ in the low-risk groups $(0.8 \%, 1.3 \%$, and $1.2 \%$ respectively). Thus, the NNT to avoid the primary outcome over a 3-year period was 27 to 30 in each of the high-risk groups versus
54 in all patients with a history of AMI and 79 to 130 in the low-risk subgroups. ${ }^{18}$ That is, in those patients who were more difficult to manage and had a higher risk of events, the reduction of $\mathrm{CV}$ risk with evolocumab was more substantial. In this context, it would be reasonable, then, to direct this type of therapy preferentially to those patients with more severe dyslipidemia, considering the more substantial reductions of LDL and, consequently, more encouraging benefits and greater cost-effectiveness.

Another aspect to be considered relates to the regression of atheroma volume. Large reductions in LDL levels can promote such an effect, as was suggested by the GLAGOV trial. ${ }^{19}$ In this experiment, 968 patients were included in 226 centers across 32 countries. Participants with symptomatic CAD were diagnosed by coronary computed tomography angiography and received monthly evolocumab $(420 \mathrm{mg})$ vs. placebo for 76 weeks, in addition to statins. At the start of the study, the mean LDL level of the participants was $93 \mathrm{mg} / \mathrm{dL}$; by the end, those randomized to evolocumab reached $29 \mathrm{mg} / \mathrm{dL}$, versus $90 \mathrm{mg} / \mathrm{dL}$ in controls. In addition, greater regression of atherosclerotic plaque was observed in the evolocumab group (64.3\% vs. $47.35 \%$; $p<0.0001)$, making GLAGOV the first study to demonstrate the benefits of PCSK9i on atherosclerotic plaque. ${ }^{19}$ These results appear to hold relevance to clinical practice, as well as external validity.

Animal studies play a fundamental role in the development of new drugs. In experiments with mice, administration of alirocumab (3 or $10 \mathrm{mg} / \mathrm{kg}$ ) for 18 weeks reduced plasma lipid levels, mitigated development of atherosclerosis and improved plaque morphology. When used in combination with atorvastatin $(3.6 \mathrm{mg} / \mathrm{kg} / \mathrm{d})$, the severity of atherosclerotic lesions was reduced even further, in a dose-dependent manner. ${ }^{20}$ However, trials with larger samples - and, preferably, in humans - are lacking.

It is estimated that 24 million patients in the U.S. alone could be eligible for PCSK9i therapy. ${ }^{21}$ Although there are no such data for the Brazilian population, the efficacy and safety of these agents have been recognized by regulatory agencies in the country, and two PCSK9i have been approved by the National Health Surveillance Agency (ANVISA) and are commercially available: Praluent ${ }^{\circledR}$ (alirocumab) and Repatha $^{\mathrm{TM}}$ (evolocumab). ${ }^{22}$ Their approved indications for use in Brazil, as well as dosages and the magnitude of LDL reduction achieved, are summarized in table 1 .

\section{General recommendations for the use of PCSK9i in clinical guidelines}

Several guidelines, including those cited in subsequent paragraphs, are unanimous in indicating the therapeutic use of PCSK9i only for those patients considered to be at high or very high risk and who were unable to reach LDL targets even after lipid-lowering therapy (such as statins at maximum tolerated dose or statins plus ezetimibe).

The UK National Institute for Health and Care Excellence (NICE) does not recommend the use of PCSK9i for patients with primary non-familial hypercholesterolemia or mixed dyslipidemia without evidence of $\mathrm{CV}$ disease, regardless of LDL concentration. In patients at high CV risk, the use of 


\section{Review Article}

\section{Table 1 - Indications for PCSK9i use in Brazil, according to the Brazilian Guideline on Dyslipidemia. ${ }^{24}$}

\begin{tabular}{|c|c|}
\hline \multicolumn{2}{|c|}{ Patients at high risk of a CV event } \\
\hline \multicolumn{2}{|c|}{ On treatment with statins at the highest tolerated dose } \\
\hline \multicolumn{2}{|c|}{ Statin or statin + ezetimibe therapy } \\
\hline \multicolumn{2}{|c|}{$\begin{array}{c}\text { Statin-intolerant or has not met recommended LDL or non-HDL goals } \\
\downarrow\end{array}$} \\
\hline Evolocumab (Repatha ${ }^{\mathrm{TM}}$ ) & Alirocumab (Praluent $囚$ ) \\
\hline $\begin{array}{l}140 \mathrm{mg} \text { by subcutaneous injection every } 2 \text { weeks or } 420 \mathrm{mg} \text { once a month } \\
\text { Both doses reduce LDL by approximately } 60 \%{ }^{25}\end{array}$ & $\begin{array}{c}75 \mathrm{mg} \text { or } 150 \mathrm{mg} \text { by subcutaneous injection every } 2 \text { weeks } \\
\text { The 75-mg and 150-mg doses are associated with average LDL reductions of } 45 \% \\
\text { and } 60 \% \text {, respectively. }{ }^{25}\end{array}$ \\
\hline
\end{tabular}

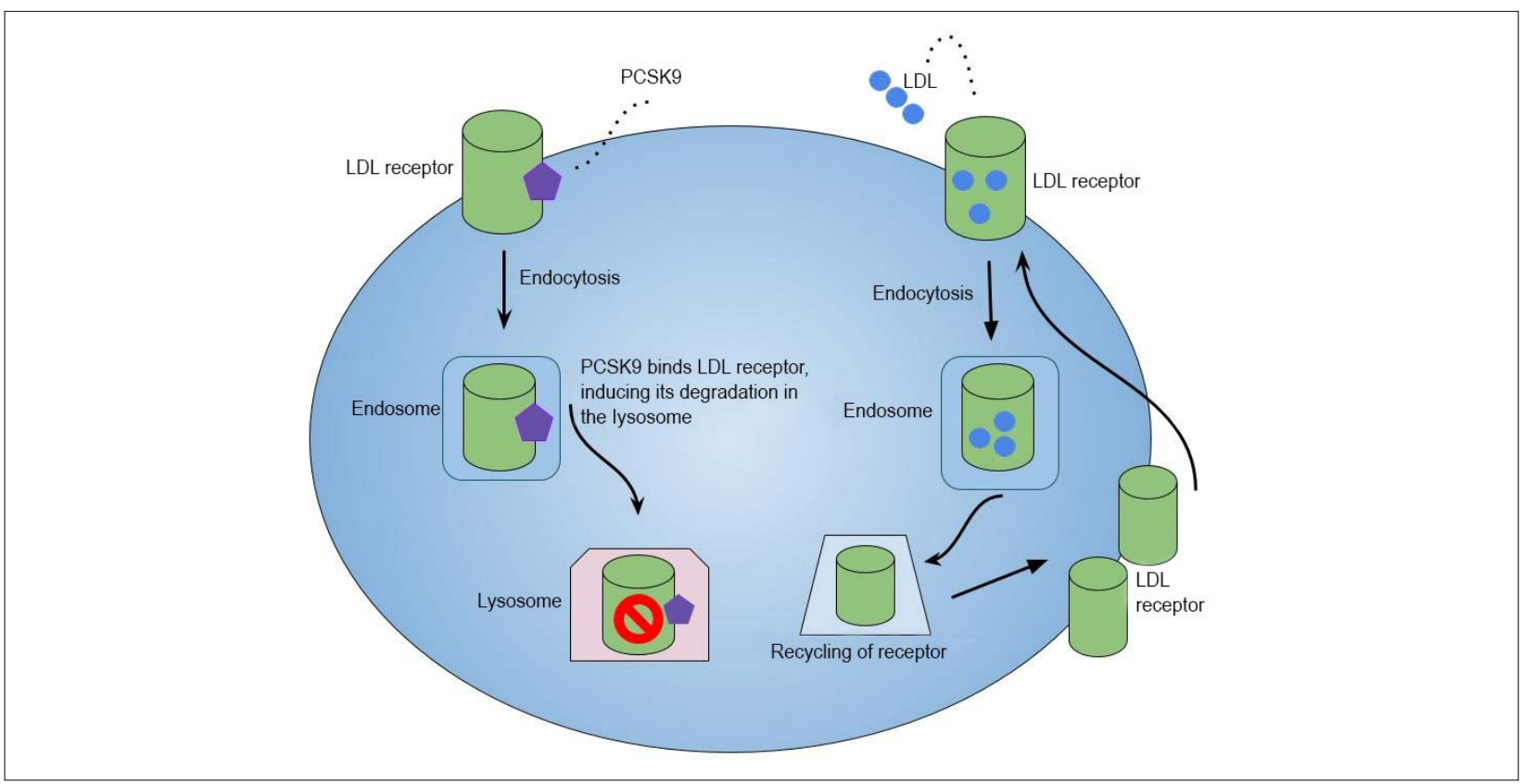

Figure 1 - Mechanisms of PCSK9 involvement in LDL metabolism.

PCSK9i is recommended only if the LDL concentration is persistently above $4.0 \mathrm{mmol} / \mathrm{L}$ (approximately $154 \mathrm{mg} / \mathrm{dL}$ ). If the patient is considered to be at very high CV risk, PCSK9i therapy is recommended only if the LDL concentration is persistently above $3.5 \mathrm{mmol} / \mathrm{L}$ (approximately $135 \mathrm{mg} / \mathrm{dL}$ ). ${ }^{23}$

In contrast, the updated Brazilian Guidelines for Dyslipidemias and Prevention of Atherosclerosis ${ }^{24}$ adopted a much less conservative addendum. In those patients at high risk for $\mathrm{CV}$ disease, the therapeutic goal of LDL should be below $70 \mathrm{mg} / \mathrm{dL}$, while in those considered at very high risk CV, the goal is to reach LDL levels below $50 \mathrm{mg} / \mathrm{dL}$. Accordingly, the 2017 consensus of the American College of Cardiology states that, for patients at higher risk (such as those with acute coronary syndrome or with multivessel CAD), a target LDL level of $<50 \mathrm{mg} / \mathrm{dL}$ can be considered. ${ }^{25}$ The American Association of Clinical Endocrinologists/American College of Endocrinology statement recommends a target $\mathrm{LDL}$ level $<55 \mathrm{mg} / \mathrm{dL}$ for: a) patients with progressive atherosclerotic CV disease; b) patients with atherosclerotic $\mathrm{CV}$ disease in association with diabetes and/or stage 3 or 4 chronic kidney disease; c) patients with heterozygous familial hypercholesterolemia (HF); and d) those with premature atherosclerotic CV disease.

In turn, the European Society of Cardiology/European Atherosclerosis Society Task Force recommends PCSK9i therapy when $\mathrm{LDL}$ is $\geq 140 \mathrm{mg} / \mathrm{dL}$ and the patient is already on combined statin and ezetimibe therapy; or when LDL is $\geq 100 \mathrm{mg} / \mathrm{dL}$ in cases of rapid progression of atherosclerotic CV disease. ${ }^{26}$ In these individuals, PCSK9i therapy is recommended with a target $\mathrm{LDL}$ level $<70 \mathrm{mg} / \mathrm{DL} .^{27}$

\section{Patients with and without diabetes mellitus}

Preclinical and clinical epidemiological studies have revealed an association of PCSK9 levels with insulin resistance and the risk of developing type 2 diabetes mellitus (DM2). ${ }^{28,29}$ Although genetic study findings have been contradictory, there seems to be a positive association between levels of PCSK9 and the incidence of DM2. ${ }^{28}$ The Dallas Heart Study found that PCSK9 levels were significantly higher in patients with DM2. ${ }^{29}$ Regular use of statins and fibrates may increase 
plasma levels of PCSK9, 30,31 with the latter potentially raising levels by up to $25 \% .^{31}$ This fact should be taken into account.

Statins themselves may also increase the incidence of DM2. A meta-analysis including more than 91,000 patients followed up for 4 years found a $9 \%$ increase in the risk of DM2 with the use of statins. ${ }^{32}$ In fact, data show that the gain in function in the LDL receptor gene is capable of impairing the insulin-secreting capacity of the pancreatic beta-cells. ${ }^{33}$ Thus, it is only natural that upregulation of LDL receptors with the use of PCSK9i might induce a decline in insulin release, thus facilitating development of new-onset DM2. Following this reasoning, a meta-analysis that evaluated short-term therapy with PCSK9i (1.5 years) found a small, but significant increase in plasma glucose and glycated hemoglobin levels. Moreover, this increase was proportional to the reduction in LDL, but was not enough to cause an impact on the emergence of new cases of DM2. ${ }^{34}$

The safety of PCSK9i therapy has also been assessed. In a pre-specified meta-analysis of the FOURIER trial, the efficacy and safety of evolocumab was investigated in patients with and without DM2, in addition to the effect of evolocumab on blood glucose and on the risk of developing DM2. ${ }^{35}$ Of those individuals already living with DM2, 8,000 had available data and $25 \%$ were on insulin. Among patients without the disease, $38 \%$ had prediabetes and $22 \%$ were normoglycemic. Both groups were homogeneous in terms of statin therapy, with $70 \%$ on maximal doses. ${ }^{35}$ Evolocumab therapy significantly reduced $\mathrm{CV}$ risk in both groups, and did not increase the risk of recent-onset DM2; there was no worsening in blood glucose levels. These data suggest that evolocumab therapy is safe and effective in patients with atherosclerotic disease. Furthermore, the number needed to prevent a primary CV event over a 3-year period among DM2 patients was only 37. Therefore, the use of PCSK9i in patients with atherosclerotic CV disease and DM2 can be particularly attractive from the point of view of cost-benefit. ${ }^{35}$

\section{Possible anti-inflammatory mechanisms and pleiotropic effects}

The potential for anti-inflammatory action by PCSK9i is unclear. Unlike therapy with statins, there is no evidence of a potential role of PCSK9i in reducing C-reactive protein (CRP) levels, especially when measured by high-sensitivity methods (hsCRP). Two recent meta-analyses that evaluated approximately 7,000 patients ${ }^{36,37}$ did not confirm this hypothesis.

Although the relationship between PCSK9 and carotid intima-media thickness in healthy patients is controversial, it may play a direct role in the inflammatory process, contributing to atherosclerotic disease through LDL-independent mechanisms. ${ }^{38}$ Whether these monoclonal antibodies interact with other pathways to induce an anti-inflammatory response is still unclear, and warrants further investigation.

The relationship between serum levels of PCSK9 and atherosclerotic plaque characteristics has also been studied. Virtual-histology intravascular ultrasound (VH-IVUS) was used to analyze 581 patients with CAD, ${ }^{39}$ and higher levels of PCSK9 were found to be associated with a greater fraction and amount of central necrotic tissue in coronary atherosclerosis, independent of LDL levels and statin therapy. ${ }^{39}$ Therefore, PCSK9 seems to play a role that goes far beyond regulation of LDL.

In another sub-analysis of the FOURIER trial, ${ }^{40}$ evolocumab acted effectively against initial inflammatory risk in 27,564 patients at high CV risk. It bears stressing that the relative benefit of therapy with this drug for the prevention of CV events was independent of baseline CRP levels. Although those patients with higher hsCRP levels exhibited greater susceptibility to CV events, they were also those who tended to derive the greatest absolute benefit from evolocumab therapy. ${ }^{40}$

Evidence suggests that vascular smooth muscle cells produce higher amounts of PCSK9 compared to endothelial cells, especially in an inflammatory microenvironment. In those regions where there is lower shear stress (i.e., force of blood friction against the arterial intima), PCSK9 expression is increased in smooth-muscle cells. Moreover, oxidized LDL appears to be implicated in the regulation of PCSK9 expression by modulating the secretion of pro-inflammatory cytokines, such as interleukin-1 (IL-1), interleukin-6 (IL-6), and tumor necrosis factor alpha (TNF- $\alpha$ ). ${ }^{41}$ Corroborating these findings, Ricci et al. ${ }^{42}$ tested the hypothesis of a relationship between PCSK9 and pro-inflammatory effects in macrophages. The authors initially performed a series of experiments with macrophages derived from the human monocytes cell line THP-1, incubated with increasing concentrations of recombinant human PCSK9. A positive correlation was observed between levels of PCSK9 and inflammatory response in macrophages, inducing expression of TNF- $\alpha$, IL-1, IL-6, as well as chemokines such as monocyte chemoattractant protein-1 (MCP-1). In addition, an inflammatory response was observed when THP-1 macrophages were co-cultured with HepG2 cells overexpressing PCSK9. ${ }^{42}$ This provides additional evidence of a pro-inflammatory effect of PCSK9.

Recently, Bernelot Moens et al. ${ }^{43}$ evaluated the responses to PCSK9i in monocytes (key mediators of the inflammatory process) of patients with $\mathrm{FH}$ who were not on statins due to muscle pain. Several pro-inflammatory and migratory alterations were observed in these monocytes. After 6 months of treatment with PCSK9i, the migratory capacity of monocytes, their lipid content, and their inflammatory responsiveness decreased to levels observed in $\mathrm{FH}$ patients on stable statin therapy. The reduction in lipid content with the use of PCSK9i attenuated the pro-inflammatory phenotype of monocytes. ${ }^{43}$ These findings are important, because they emphasize that other mediators beyond CRP are involved in inflammation.

Finally, it should be emphasized that the PCSK9i agents have pleiotropic effects, and that their use may have other therapeutic actions, in addition to their already-established hypolipidemic activity.

\section{Safety}

In 2012, the U.S. Food and Drug Administration (FDA) issued an alert on the potential adverse effects of statin treatment. ${ }^{44}$ Two years later, the FDA asked PCSK9i developers to assess possible adverse events of these drugs in different studies, with special attention to the emergence of new cases of cognitive deficit. ${ }^{45}$ This recommendation was based on 


\section{Review Article}

some reports that warned of a possible increase in the risk of neurocognitive events with the use of PCSK9i. Indeed, there is some biological plausibility to support the argument that a very sharp reduction in lipids can negatively impact cognitive function, regardless of the ability of the drug to cross the blood-brain barrier. ${ }^{46,47}$

To date, the leading assessment of the risk of cognitive deficits with the use of a PCSK9i (evolocumab) plus statin as compared to placebo plus statin is the EBBINGHAUS trial, ${ }^{48}$ which randomized 1,974 patients. The subjects had a mean age of 63 years and were followed up for approximately 19 months. All completed the Cambridge Neuropsychological Test Automated Battery at 6, 12, and 24 months. No difference was observed between the groups in terms of cognitive function, scores on the cognitive function battery, or in subjective self-assessment of daily cognitive ability. ${ }^{48}$

In a pre-specified secondary analysis of the FOURIER study, Giugliano et al. ${ }^{49}$ analyzed approximately 26,000 patients, with special attention to the relationship between the LDL concentration reached at 4 weeks and subsequent CV outcomes. There was no reduction in safety with very low LDL concentrations over an average of 2 years.

In the MENDEL-2 ${ }^{50}$ study, a large trial of evolocumab monotherapy, there was a rapid and marked decrease in levels of LDL and apolipoprotein B over 12 weeks in comparison with the placebo or ezetimibe group. LDL reductions in excess of $50 \%$ were reported in $72 \%$ of patients who received evolocumab. Severe adverse effects occurred at comparable rates across groups. In addition, injection-site reactions were infrequent with evolocumab and did not differ between groups. Biweekly and monthly evolocumab administration yielded comparable reductions in LDL levels, with good tolerability and safety. ${ }^{50}$

The LAPLACE- $2^{51}$ study compared evolocumab versus ezetimibe versus placebo in patients with hypercholesterolemia who were receiving stable doses of statins. Adverse events were similar in the three groups (36\% of patients treated with statin plus evolocumab, $40 \%$ of those who received statin plus ezetimibe, and $39 \%$ of those who received statin plus placebo); musculoskeletal symptoms and headache were the most common. Intolerable adverse events that resulted in discontinuation of treatment occurred in only $1.9 \%, 1.8 \%$, and $2.2 \%$ of participants in the evolocumab, ezetimibe, and placebo groups, respectively. Severe adverse events were reported in $2.1 \%$ of the patients treated with evolocumab, $0.9 \%$ of those treated with ezetimibe, and $2.3 \%$ of those in the control group. Neurocognitive events were reported in only 1 patient treated with evolocumab, compared with 3 patients treated with ezetimibe and no patients in the control groups. It bears stressing that the study was conducted for a short period (3 months) and, despite some adverse events, the benefits seemed to outweigh the risk of PCSK9i therapy. ${ }^{51}$

The GAUSS-2 ${ }^{52}$ study evaluated evolocumab versus ezetimibe in statin-intolerant dyslipidemic patients over 3 months. The rate of adverse events leading to treatment discontinuation was $8 \%$ in the evolocumab group - lower than in the ezetimibe arm (13\%). Muscle pain occurred in only $8 \%$ of patients treated with evolocumab, versus $18 \%$ of those treated with ezetimibe. Discontinuation due to musculoskeletal side effects occurred in 5\% of patients the evolocumab group, again a rate numerically lower than in the ezetimibe group (6\%). ${ }^{52}$ In the GAUSS-3 study, ${ }^{53}$ patients who were intolerant to statins were treated with evolocumab $420 \mathrm{mg}$ (with placebo ezetimibe) or ezetimibe $10 \mathrm{mg}$ per day (with placebo evolocumab). Myalgia was reported by approximately $29 \%$ of patients treated with ezetimibe and $21 \%$ of those treated with evolocumab. However, muscular symptoms leading to discontinuation were very infrequent in the evolocumab group, occurring in only 1 out of 145 treated patients. ${ }^{53}$ This seems very relevant, as it suggests that PCSK9i therapy can be used successfully in people with statin intolerance.

Interestingly, subjects with null mutation of the PCSK9 gene have been described. A U.S. woman inherited a mutation from her father and another from her mother which effectively eliminated PCSK9 function. ${ }^{54}$ Her lifetime average LDL levels were only $14 \mathrm{mg} / \mathrm{dL}$ and, more importantly, she seems to lead a healthy life. In other words, even in a setting of marked reduction of LDL to extraordinarily low levels due to a genetic mutation, there is no evidence of any relevant harm to the overall health of the individual.

Another factor that is worthy of note is measurement of vitamin $\mathrm{E}$ levels. It is known that lipoproteins are involved in vitamin $\mathrm{E}$ transport, ${ }^{55}$ and are necessary for steroidogenesis. Therefore, when levels of LDL are extremely low, vitamin E measurement - and, possibly, supplementation - seems necessary. ${ }^{56}$ In fact, data from the DESCARTES ${ }^{57}$ study showed that the substantial reduction in LDL in patients treated with evolocumab also reduced their levels of vitamin E. Nevertheless, there was no alteration in tissue levels of vitamin $\mathrm{E}$, and the reduction was not clinically significant. Furthermore, there is no evidence of compromised synthesis of steroid, adrenal, or gonadal hormones, even in patients with extremely low LDL. ${ }^{57}$ Overall, these data support that even very low concentrations of LDL by inhibition of PCSK9 do not translate into increased risk. In addition to these results, preliminary data from an analysis of nearly 3,000 patients enrolled in the DESCARTES and OSLER-1 studies showed no increase in adverse events and no cases of hemorrhagic stroke among patients with LDL levels below 40 or $25 \mathrm{mg} / \mathrm{dL} .{ }^{50}$

Two open-label extensions of the FOURIER study, designed to evaluate the long-term safety of evolocumab in approximately 6,600 patients, are underway. ${ }^{49}$ These results will certainly provide clearer evidence on the safety profile of PCSK9i.

\section{Cost-effectiveness}

Despite current evidence supporting the superiority of PCSK9 $i$ in the reduction of LDL concentrations in comparison to statins and ezetimibe, the cost-effectiveness ratio cannot be ignored. Estimates suggest that use of these agents is associated with significant expenditures for patients in different scenarios: a) $€ 78,485.00$ for those with a family history of hypercholesterolemia alone; b) $€ 176,735.00$ for those with 
10-year CV risk > 30\%; and c) €295,543.00 for patients with established CV disease and DM2, all per quality-adjusted life-year (QALY) gained. ${ }^{58}$ Additionally, the estimated annual cost of treatment is US\$14,000.00. ${ }^{59}$ This becomes particularly important when considering the (implicit and estimated) willingness-to-pay threshold in Brazilian, which seems to fluctuate between $\mathrm{R} \$ 25,000.00$ and $\mathrm{R} \$ 185,000.00$ per QALY ${ }^{60}$ Cost-effectiveness ratio and cost-utility ratio estimates indicate values much higher than the Brazilian thresholds, and, at least for the prospect of third-party payers, these drugs will have to spend some time on the market before their prices decline enough for consideration. According to Moore's curve, when a new technology is incorporated, there is a non-negligible gap in time for a reduction in estimated price (e.g., $60 \%$ to $70 \%$ of the current value). ${ }^{61-63}$ Therefore, it is important to evaluate the cost-effectiveness and cost-utility of PCSK9i and their market prices before they can be recommended as a therapeutic option - at this time, still from the patient perspective alone.

\section{Final considerations}

Since the discovery of their effect on LDL levels, PCSK9i have been an object of great research interest. The clear association between $\mathrm{CV}$ risk factors and the significant reduction in LDL obtained with their use are guiding the development of novel algorithms for the treatment of dyslipidemias and $\mathrm{CV}$ diseases as a whole.

Major advances in the treatment of CAD have been achieved in recent decades. Among them, the greater understanding of the importance of LDL as a causal factor was particularly relevant. The results of the RCTs described in this paper have provided the evidence base for the use of PCSK9i and indications for the use of these drugs, as well as the LDL targets to be achieved. Each patient should have their risk adequately assessed, taking into account the cost-effectiveness of treatment and the most appropriate medications for their clinical condition. The inhibition of PCSK9 represents a novel approach to reducing LDL levels and preventing adverse

\section{References}

1. Schlatter RP, Hirakata VN, Polanczyk CA. Estimating the direct costs of ischemic heart disease: evidence from a teaching hospital in BRAZIL, a retrospective cohort study. BMC Cardiovasc Disord. 2017;17(1):180.

2. Ballantyne CM. Low-density lipoproteins and risk for coronary artery disease. Am J Cardiol. 1998;82(9A):3Q-12Q.

3. Chaudhary R, Mathew D, Bliden K, Tantry US, Sharma T, Gesheff MG, et al. Low-density lipoprotein 4 : a novel predictor of coronary artery disease severity. Curr Med Res Opin. 2017;33(11):1979-84.

4. Khera AV, Kathiresan S. Genetics of coronary artery disease: discovery, biology and clinical translation. Nat Rev Genet. 2017;18(6):331-44.

5. Kopin L, Lowenstein C. Dyslipidemia. Ann Intern Med. 2017; 167(11):ITC81-ITC96.

6. Sabatine MS. Advances in the treatment of dyslipidemia. Cleve Clin J Med. 2016;83(3):181-6.
$\mathrm{CV}$ outcomes in high-risk patients who have not achieved recommended levels of LDL despite the use of a maximally optimized therapeutic arsenal.

Finally, it is important to stress that the use of PCSK9i should not be indiscriminate, and that it is up to physicians to determine which patients will actually benefit from their use. On the other hand, high-risk patients and those who are intolerant to statins - and who can afford this therapy - certainly have in PCSK9i a treatment option that has so far proven to be safe and attractive.

\section{Acknowledgements}

Financial support was provided by the Hospital de Clínicas de Porto Alegre Research and Event Incentive Fund (FIPE-HCPA).

\section{Author contributions}

Conception and design of the research: Ferrari $F$, Moriguchi EH; acquisition of data, analysis and interpretation of the data and writing of the manuscript: Ferrari F, Stein R, Motta MT, Moriguchi EH; critical revision of the manuscript for intellectual content: Stein R, Moriguchi EH.

\section{Potential Conflict of Interest}

No potential conflict of interest relevant to this article was reported.

\section{Sources of Funding}

There were no external funding sources for this study.

\section{Study Association}

This study is not associated with any thesis or dissertation work.

\section{Ethical approval and informed consent}

This article does not report on human or animal studies by any of the authors.

7. Rodriguez F, Harrington RA. Cholesterol, cardiovascular risk, statins, PCSK9 inhibitors, and the future of LDL-C lowering. JAMA. 2016;316(19):1967-8.

8. Sabatine MS, Giugliano RP, Keech AC, Honarpour N, Wiviott SD, Murphy $\mathrm{SA}$, et al. Evolocumab and clinical outcomes in patients with cardiovascular disease. N Engl J Med. 2017;376(18):1713-22.

9. Landmesser U, Chapman MJ, Stock JK, Amarenco P, Belch JJF, Borén J, et al. 2017 Update of ESC/EAS Task Force on practical clinical guidance for proprotein convertase subtilisin/kexin type 9 inhibition in patients with atherosclerotic cardiovascular disease or in familial hypercholesterolaemia. Eur Heart J. 2018;39(14):1131-43.

10. Faludi AA, Izar MCO, Saraiva JFK, Chacra APM, Bianco HT, Afiune A Neto, et al. Atualização da Diretriz Brasileira de Dislipidemias e Prevenção da Aterosclerose - 2017. Arq Bras Cardiol. 2017;109(2Supl.1):1-76.

11. Chaudhary R, GargJ, Shah N, Sumner A. PCSK9 inhibitors: a new era of lipid lowering therapy. World J Cardiol. 2017;9(2):76-91. 
12. Stein EA, Raal F. Reduction of low-density lipoprotein cholesterol by monoclonal antibody inhibition of PCSK9. Annu Rev Med. 2014;65:417-31.

13. Schmidt AF, Pearce LS, Wilkins JT, Overington JP, Hingorani AD, Casas JP. PCSK9 monoclonal antibodies for the primary and secondary prevention of cardiovascular disease. Cochrane Database Syst Rev. 2017 Apr 28;4:CD011748.

14. Ridker PM, Danielson E, Fonseca FA, Genest J, Gotto AM Jr, Kastelein JJ, et al. Rosuvastatin to prevent vascular events in men and women with elevated C-reactive protein. N Engl J Med. 2008;359(21):2195-207.

15. Stossel TP. The discovery of statins. Cell. 2008;134(6):903-5.

16. American College of Cardiology. ODYSSEY Outcomes: Results Suggest Use of PCSK9 Inhibitor Reduces CV Events, LDL-C in ACS Patients [Internet]. Washington DC: American College of Cardiology; 2018. [citado 15 mar. 2018]. Disponível em: https://www.acc.org/latest-in-cardiology/ articles/2018/03/05/15/53/sat-9am-odyssey-outcomes-cv-outcomes-withalirocumab-after-acs-acc-2018.

17. Cholesterol Treatment Trialists' (CTT) Collaboration, Baigent C, Blackwell L, Emberson J, Holland LE, Reith C, et al. Efficacy and safety of more intensive lowering of LDL cholesterol: a meta-analysis of data from 170,000 participants in 26 randomised trials. Lancet. 2010;376(9753):1670-81.

18. Sabatine MS, De Ferrari GM, Giugliano RP, Huber K, Lewis BS, Ferreira J, et al. Clinical benefit of evolocumab by severity and extent of coronary artery disease. Circulation. 2018;138(8):756-766.

19. Nicholls SI, Puri R, Anderson T, Ballantyne CM, Cho L, Kastelein IJ, et al. Effect of evolocumabe on progression of coronary disease in statin-treated patients: The GLAGOV randomized clinical trial. JAMA. 2016;316(22):2373-84.

20. Kühnast S, van der Hoorn JW, Pieterman EJ, van den Hoek AM, Sasiela WJ, Gusarova V, et al. Alirocumabe inhibits atherosclerosis, improves the plaque morphology, and enhances the effects of a statin. J Lipid Res. 2014;55(10):2103-12.

21. Choi J, Khan AM, Jarmin M, Goldenberg N, Glueck CJ, Wang P. Efficacy and safety of proprotein convertase subtilisin-kexin type 9 (PCSK9) inhibitors, alirocumabe and evolocumabe, a post-commercialization study. Lipids Health Dis. 2017;16(1):141.

22. Weintraub WS, Gidding SS. PCSK9 inhibitors: a technology worth paying for? Pharmacoeconomics. 2016;34(3):217-20.

23. Mayor S. NICE recommends PCSK9 inhibitors for patients not responding to statins. BMJ. 2016 May 8;353:i2609.

24. Faludi AA, Izar MCO, Saraiva JFK, Chacra APM, Bianco HT, Afiune A Neto, et al. Atualização da Diretriz Brasileira de Dislipidemias e Prevenção da Aterosclerose - 2017. Arq Bras Cardiol. 2017;109(2 Supl.1):1-76.

25. Rosenson RS, Hegele RA, Fazio S, Cannon CP. The evolving future of PCSK9 inhibitors. J Am Coll Cardiol. 2018;72(3):314-29.

26. Baum SJ, Toth PP, Underberg JA, Jellinger P, Ross J, Wilemon K. PCSK9 inhibitor access barriers-issues and recommendations: improving the access process for patients, clinicians and payers. Clin Cardiol. 2017;40(4):243-54.

27. Landmesser U, John Chapman M, Farnier M, Gencer B, Gielen S, Hovingh GK, et al. European Society of Cardiology/European Atherosclerosis Society Task Force consensus statement on proprotein convertase subtilisin/ kexin type 9 inhibitors: practical guidance for use in patients at very high cardiovascular risk. Eur Heart J. 2017;38(29):2245-55.

28. Momtazi AA, Banach M, Pirro M, Stein EA, Sahebkar A. PCSK9 and diabetes: is there a link? Drug Discov Today. 2017;22(6):883-95.

29. Lakoski SG, Lagace TA, Cohen JC, Horton JD, Hobbs HH. Genetic and metabolic determinants of plasma PCSK9 levels. J Clin Endocrinol Metab. 2009;94(7):2537-43.
30. Welder G, Zineh I, Pacanowski MA, Troutt JS, Cao G, Konrad RJ. High-dose atorvastatin causes a rapid sustained increase in human serum PCSK9 and disrupts its correlation with LDL cholesterol. J Lipid Res. 2010;51(9):2714-21.

31. Konrad RJ, Troutt JS, Cao G. Effects of currently prescribed LDL-C-lowering drugs on PCSK9 and implications for the next generation of LDL-C-lowering agents. Lipids Health Dis. 2011 Feb 28;10:38.

32. Sattar N, Preiss D, Murray HM, Welsh P, Buckley BM, de Craen AJ, et al. Statins and risk of incident diabetes: a collaborative meta-analysis of randomised statin trials. Lancet. 2010;375(9716):735-42.

33. Roehrich ME, Mooser V, Lenain V, Herz J, Nimpf J, Azhar S, et al. Insulinsecreting beta-cell dysfunction induced by human lipoproteins. J Biol Chem. 2003;278(20):18368-75.

34. de Carvalho LSF, Campos AM, Sposito AC. Proprotein convertase subtilisin/ kexin type 9 (PCSK9) inhibitors and incident type 2 diabetes: a systematic review and meta-analysis with over 96,000 patient-years. Diabetes Care. 2018;41(2):364-67.

35. Sabatine MS, Leiter LA, Wiviott SD, Giugliano RP, Deedwania P, De Ferrari GM, et al. Cardiovascular safety and efficacy of the PCSK9 inhibitor evolocumabe in patients with and without diabetes and the effect of evolocumabe on glycaemia and risk of new-onset diabetes: a prespecified analysis of the FOURIER randomised controlled trial. Lancet Diabetes Endocrinol. 2017;5(12):941-50.

36. Sahebkar A, Di Giosia P, Stamerra CA, Grassi D, Pedone C, Ferretti G, et al. Effect of monoclonal antibodies to PCSK9 on high-sensitivity C-reactive protein levels: a meta-analysis of 16 randomized controlled treatment arms. BrJ Clin Pharmacol. 2016;81(6):1175-90.

37. Cao YX, Li S, Liu HH, Li JJ. Impact of PCSK9 monoclonal antibodies on circulating hs-CRP levels: a systematic review and meta-analysis of randomised controlled trials. BMJ Open. 2018;8(9):e022348.

38. Urban D, Poss J, Bohm M, Laufs U. Targeting the proprotein convertase subtilisin/kexin type 9 for the treatment of dyslipidemia and atherosclerosis. J Am Coll Cardiol. 2013;62(16):1401-8.

39. Cheng JM, Oemrawsingh RM, Garcia-Garcia HM, Boersma E, van Geuns RJ, Serruys PW, et al. PCSK9 in relation to coronary plaque inflammation: results of the ATHEROREMO-IVUS study. Atherosclerosis. 2016 May;248:117-22.

40. Bohula EA, Giugliano RP, Leiter LA, Verma S, Park JG, Sever OS, et al. Inflammatory and cholesterol risk in the FOURIER trial. Circulation. 2018;138(2):131-40.

41. Liberale L, Montecucco F, Camici GG, Dallegri F, Vecchie A, Carbone F, et al. Treatment with proprotein convertase subtilisin/kexin type 9 (PCSK9) inhibitors to reduce cardiovascular inflammation and outcomes. Curr Med Chem. 2017;24(14):1403-16.

42. Ricci C, Ruscica M, Camera M, Rossetti L, Macchi C, Colciago A, et al. PCSK9 induces a pro-inflammatory response in macrophages. Sci Rep. 2018;8(1):2267.

43. Bernelot Moens SJ, Neele AE, Kroon J, van der Valk FM, Van den Bossche J, Hoeksema MA, et al. PCSK9 monoclonal antibodies reverse the proinflammatory profile of monocytes in familial hypercholesterolaemia. Eur Heart J. 2017;38(20):1584-93.

44. Mefford MT, Rosenson RS, Cushman M, Farkouh ME, McClure LA, Wadley VG, et al. PCSK9 variants, low-density lipoprotein cholesterol, and neurocognitive impairment: reasons for geographic and racial differences in stroke study (REGARDS). Circulation. 2018;137(12):1260-69.

45. Harvey PD, Sabbagh MN, Harrison JE, Ginsberg HN, Chapman MJ, Manvelian G, et al. No evidence of neurocognitive adverse events associated with alirocumab treatment in 3340 patients from 14 randomized Phase 2 and 3 controlled trials: a meta-analysis of individual patient data. Eur Heart J. 2018;39(5):374-81. 
46. Ott BR, Daiello LA, Dahabreh IJ, Springate BA, Bixby K, Murali M, et al. Do statins impair cognition? a systematic review and meta-analysis of randomized controlled trials. J Gen Intern Med. 2015;30(3):348-58

47. Khan AR, Bavishi C, Riaz H, Farid TA, Khan S, Atlas M, et al. Increased risk of adverse neurocognitive outcomes with proprotein convertase subtilisin-kexin type 9 inhibitors. Circ Cardiovasc Qual Outcomes. 2017;10(1):pii:e003153.

48. Giugliano RP, Mach F, Zavitz K, Kurtz C, Im K, Kanevsky E, et al Cognitive function in a randomized trial of evolocumab. N Engl J Med. 2017;377(7):633-43.

49. Giugliano RP, Pedersen TR, Park JG, De Ferrari GM, Gaciong ZA, Ceska $R$, et al. Clinical efficacy and safety of achieving very low LDL-cholesterol concentrations with the PCSK9 inhibitor evolocumab: a prespecified secondary analysis of the FOURIER trial. Lancet. 2017;390(10106):1962-71.

50. Koren MJ, Lundqvist P, Bolognese M, Neutel JM, Monsalvo ML, Yang J, et al. Anti-PCSK9 monotherapy for hypercholesterolemia: the MENDEL-2 randomized, controlled phase III clinical trial of evolocumab. J Am Coll Cardiol. 2014;63(23):2531-40.

51. Robinson JG, Nedergaard BS, Rogers WJ, Fialkow J, Neutel JM, Ramstad D, et al. Effect of evolocumab or ezetimibe added to moderate- or high-intensity statin therapy on LDL-C lowering in patients with hypercholesterolemia: the LAPLACE-2 randomized clinical trial. JAMA. 2014;311(18):1870-82.

52. Stroes E, Colquhoun D, Sullivan D, Civeira F, Rosenson RS, Watts GF, et al. Anti-PCSK9 antibody effectively lowers cholesterol in patients with statin intolerance: the GAUSS-2 randomized, placebo-controlled phase 3 clinical trial of evolocumab. J Am Coll Cardiol. 2014;63(23):2541-8.

53. Nissen SE, Stroes E, Dent-Acosta RE, Rosenson RS, Lehman SJ, Sattar N, et al. Efficacy and tolerability of evolocumab vs ezetimibe in patients with muscle-related statin intolerance: the GAUSS-3 randomized clinical trial. JAMA. 2016;315(15):1580-90.

54. Hall SS. Genetics: a gene of rare effect: a mutation that gives people rockbottom cholesterol levels has led geneticists to what could be the next blockbuster heart drug. Nature [periódicos na Internet]. 2013 [acesso 16 apr 2018];496(7444). Disponível em: https://www.nature.com/news/geneticsa-gene-of-rare-effect-1.12773.

55. Hacquebard M, Carpentier YA. Vitamin E: absorption, plasma transport and cell uptake. Curr Opin Clin Nutr Metab Care. 2005;8(2):133-8.

56. Qamar A, Bhatt DL. Effect of low cholesterol on steroid hormones and vitamin E levels: just a theory or real concern? Circ Res. 2015;117(8):662-4.

57. Blom DJ, Djedjos CS, Monsalvo ML, Bridges I, Wasserman SM, Scott R, et al. Effects of evolocumab on vitamin $E$ and steroid hormone levels: results from the 52-Week, phase 3, double-blind, randomized, placebo-controlled DESCARTES study. Circ Res. 2015;117(8):731-41.

58. Stam-Slob MC, van der Graaf Y, de Boer A, Greving JP, Visseren FLJ. Costeffectiveness of PCSK9 inhibition in addition to standard lipid-lowering therapy in patients at high risk for vascular disease. Int J Cardiol. 2018 Feb $15 ; 253: 148-54$

59. McDonagh M, Peterson K, Holzhammer B, Fazio S. A systematic review of PCSK9 inhibitors alirocumab and evolocumab. J Manag Care Spec Pharm. 2016;22(6):641-653q.

60. Soarez PCD, Novaes HMD. Cost-effectiveness thresholds and the Brazilian Unified National Health System. Cad. Saude Publica. 2017;33(4):e00040717.

61. Kazi DS, Penko J, Coxson PG, Moran AE, Ollendorf DA, Tice JA, et al. Updated cost-effectiveness analysis of PCSK9 inhibitors based on the results of the FOURIER trial. JAMA. 2017;318(8):748-50.

62. Fonarow GC, Keech AC, Pedersen TR, Giugliano RP, Sever PS, Lindgren P, et al. Cost-effectiveness of Evolocumab Therapy for reducing cardiovascular events in patients with atherosclerotic cardiovascular disease. JAMA Cardiol. 2017;2(10):1069-78

63. Arrieta A, Hong JC, Khera R, Virani SS, Krumholz HM, Nasir K. Updated cost-effectiveness assessments of PCSK9 inhibitors from the perspectives of the health system and private payers: insights derived from the FOURIER trial. JAMA Cardiol. 2017;2(12):1369-74. 